The authors propose that the $A P O E^{*} 4$ allele could be an additional risk factor in CJD.

Original article Krasnianski A et al. (2007) Increased frequency of positive family history of dementia in sporadic CJD.

Neurobiol Aging [doi:10.1016/j.neurobiolaging.2007.07.014]

\section{No restrictive valvular heart disease observed in 90 PD patients on low pergolide doses}

A high incidence $(\sim 33 \%)$ of restrictive valvular heart disease (VHD) has been reported in patients with Parkinson's disease (PD) receiving the ergot dopamine agonist pergolide. As a result of such observations, the therapeutic use of pergolide in PD has been restricted. Interestingly, however, a recent Czech study has demonstrated a low incidence of VHD in PD patients treated with only moderate doses of pergolide.

The study evaluated 90 patients with PD (mean age 60.8 years; mean disease duration 10.0 years) who were receiving a mean of $2.93 \mathrm{mg} /$ day pergolide (range $0.75-5.0 \mathrm{mg} /$ day). Mean duration of pergolide therapy was 51 months, and 79 patients were also receiving levodopa. No cases of restrictive VHD or severe valve insufficiency were found on echocardiography. Mild mitral regurgitation was observed in 40 (44\%) patients, and in 13 (36\%) of 36 control individuals. One patient had a moderate mitral regurgitation. Neither duration of pergolide treatment nor cumulative pergolide dose predicted mitral valve insufficiency. Discrete fibrotic changes on valves were observed in $10(11 \%)$ patients, and degenerative changes were seen in 11 (12\%) patients (compared with $19 \%$ of control individuals).

The authors attribute the absence of observed restrictive VHD to the relatively low doses of pergolide administered to the participants in their study. They suggest that pergolide doses not exceeding $3 \mathrm{mg} /$ day could be considered for patients with $\mathrm{PD}$, as long as these individuals undergo regular echocardiographic follow-up.

Original article Rủžička E et al. (2007) Low incidence of restrictive valvulopathy in patients with Parkinson's disease on moderate doses of pergolide. J Neurol [doi:10.1007/ s00415-007-0592-x]

\section{Passive smoking increases the risk of multiple sclerosis in children}

Smoking is associated with the risk of developing multiple sclerosis (MS) in adults, but the effects of passive smoking in children are unclear. To address this issue, Mikaeloff et al. studied children with MS and matched controls to investigate whether exposure to parental smoking in the home increased the risk of a first episode of MS before 16 years of age.

This study enrolled patients with confirmed MS who had experienced a first clinical episode before the age of 16 years and at least one relapse. Each case was matched on the basis of age, sex, and current area of residence with 12 controls from the French general population. Participants were each sent a questionnaire about environmental risk factors, incidence of familial autoimmune diseases, socioeconomic status, and whether one or both parents had ever smoked within the home before the index date (i.e. onset of the first episode of MS in case patients).

The frequency of exposure to parental smoking in the home was $62.0 \%$ for the 129 case patients and $45.1 \%$ for the 1,038 controls. Exposed children had a higher risk of a first episode of MS than did those who were not exposed (rate ratio [RR] 2.09); this effect persisted after adjustment for socioeconomic status and for familial MS or other familial autoimmune diseases (RR 2.12, 95\% Cl 1.43-3.15). The adjusted risk was higher in older children ( $\geq 10$ years of age), who had a relatively long duration of exposure to cigarette smoke, than in younger individuals (RRs 2.49 and 1.47 , respectively).

The authors conclude that exposure to parental smoking increases the risk of a first episode of MS before the age of 16 years, and that this risk increases with increasing duration of exposure.

Original article Mikaeloff $Y$ et al. (2007) Parental smoking at home and the risk of childhood-onset multiple sclerosis in children. Brain 130: 2589-2595 